\title{
Research on Gearbox Fault Diagnosis Based on PCA and AGA-BP Neural Network
}

\author{
Fengyun XIE ${ }^{1}$, Jiankun DONG, Shaoshi YAN, Yongqi JIANG, and Yu FU \\ School of Mechatronics and Vehicle Engineering, East China Jiaotong University, \\ Jiangxi, Nanchang 330013, China
}

\begin{abstract}
As the key component of high-speed train bogie, the fault characteristics of gearbox are mainly reflected in its vibration signal. The vibration signals collected in the process of gearbox fault diagnosis are usually complex and changeable, and have strong randomness and contingency. A gearbox fault diagnosis method based on multi feature extraction, principal component analysis (PCA) and adaptive genetic algorithm is proposed to optimize the back propagation neural network analysis. The original vibration data in the gearbox fault diagnosis experiment published by Jiangsu qianpeng Diagnostic Engineering Co., Ltd. is extracted by multi eigenvalues. The feature set is reduced by PCA. The selected principal components are diagnosed and analyzed by AGA-BP neural network. The final diagnosis result is that the root mean square error (MSE) of AGA-BP neural network is 0.0116 , and the recognition rate of gearbox fault is $100 \%$.
\end{abstract}

Keywords. Gearbox fault recognition, feature extraction, principal component analysis, adaptive genetic algorithm, AGA-BP neural network

\section{Introduction}

Nowadays, with the rapid development and popularization of high-speed train in China, its safety performance has been paid great attention. As a key component of high-speed train running gear, its running state affects the safety of high-speed train. Therefore, it is of great significance to study the fault diagnosis method of gearbox.

The state monitoring and fault diagnosis of gearbox began in the 1980s, mainly relying on the time-frequency domain signal analysis of equipment vibration in the gearbox. Many advanced gear signal analyzers and systems have emerged, which makes the fault diagnosis of gearbox more scientific and accurate [1]. With the rapid development of artificial intelligence and feature sensor technology in recent years, the fault detection technology of gearbox has entered a new stage, which relies on expert system and artificial intelligence [2]. BP neural network is one of the most widely used and successful artificial neural networks in the field of fault diagnosis [3]. However, BP algorithm still has many shortcomings, such as long learning time, slow convergence speed and so on [4], so sometimes there is a lack of fault diagnosis effect.

${ }^{1}$ Corresponding author. Tel.:18279159474. School of Mechatronics and Vehicle Eengineering, East China Jiaotong University, Jiangxi, Nanchang 330013, China E-mail address: 1040449239@qq.com 
In this paper, taking vibration data of gearbox fault diagnosis experimental platform as samples, AGA-BP neural network model is established by using adaptive genetic algorithm and BP neural network to identify gearbox fault. The diagnosis results show that the model can diagnose the gearbox fault very well.

\section{Fundamental Theory}

\subsection{BP Neural Network}

Artificial neural network (ANN) is an adaptive nonlinear dynamic information processing system. The weights of the network can be changed through sample learning training, so that the actual output and expected output can meet the specified error requirements [5].Therefore, neural network model can be widely used in the field of fault diagnosis. BP neural network has a strong ability of nonlinear mapping. Theoretically, for a three-layer or more than three-layer BP neural network, as long as the hidden layer contains enough neuron nodes, the network can approach a nonlinear function with arbitrary precision, so it is more suitable for solving some complex problems [6] . However, BP neural network algorithm needs to optimize the very complex objective function, and the learning speed of BP network is relatively fixed. Therefore, the network needs longer training and learning time, more iterations and slower convergence speed. In view of the above problems, the neural network is improved. Through the analysis of adaptive genetic algorithm (AGA), the combination of the two algorithms can effectively improve the performance of BP networkms.

As shown in Figure 1, it is a three-layer BP neural network structure with input layer, implied layer and output layer being one layer. The neurons between layers are connected in a full way, but there is no mutual connection between the same layer neurons.

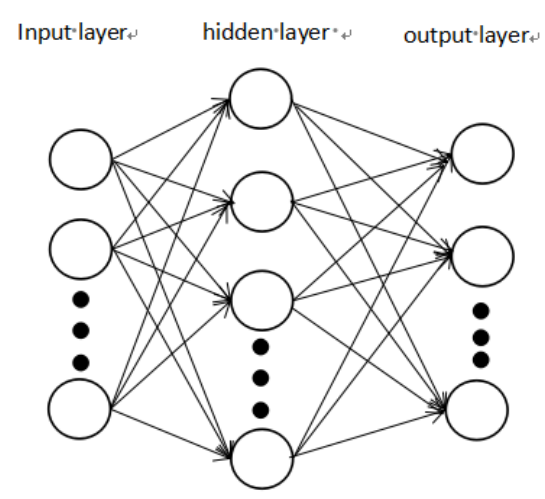

Figure 1. Structure diagram of BP neural model

\subsection{Adaptive Genetic Algorithm}

Genetic algorithm is a powerful tool to imitate the natural principle and optimize the modeling function through computer reform. Because of its powerful function, it can be combined with a variety of algorithms, and can improve the efficiency and 
feasibility of the algorithm, so it is widely used [7]. GA is a good tool to solve complex optimization problems, especially for fault diagnosis. However, the widely used standard genetic algorithm (SGA) uses fixed crossover rate and mutation rate, which leads to its premature convergence and slow convergence speed. Moreover, for some specific problems, it is often unable to find the appropriate crossover rate and mutation rate, so that the problem can not be well solved.

AGA aims to achieve the tradeoff between search and randomness in different ways, and adaptively change the values of cross rate $(\mathrm{Pc})$ and mutation rate $(\mathrm{Pm})$ according to fitness value, that is, when the current population is in the local best position, the values of $\mathrm{Pc}$ and $\mathrm{Pm}$ increase, and when the population adaptability is scattered and diversity is relatively high, the values of both decrease accordingly. The Pc and Pm of AGA are adjusted by formula (1) and (2).

$$
\begin{gathered}
P c= \begin{cases}k_{1} \frac{f_{\max }-f^{\prime}}{f_{\max }-f_{\text {avg }}} & f^{\prime} \geq f_{\text {avg }} \\
k_{2} & f^{\prime}<f_{\text {avg }}\end{cases} \\
P m= \begin{cases}k_{3} \frac{f_{\max }-f^{\prime}}{f_{\max }-f_{\text {avg }}} & f^{\prime} \geq f_{\text {avg }} \\
k_{4} & f^{\prime}<f_{\text {avg }}\end{cases}
\end{gathered}
$$

Where $f^{\prime}$ is the fitness value of the individual with larger fitness among the two cross individuals, $f_{\max }$ is the maximum fitness value in the population, $f_{\text {avg }}$ is the average fitness value, and the coefficients $K_{1}, K_{2}, K_{3}$ and $K_{4}$ are the upper values of the interval $(0,1)$.

\subsection{BP Network Optimized by Adaptive Genetic Algorithm}

In the process of BP neural network training, the connection weights and thresholds of each layer are randomly assigned, and the selection of connection weights and thresholds will affect the final prediction accuracy [8] . In other words, selecting good weights and thresholds can improve the prediction accuracy of BP neural network, and adaptive genetic algorithm is a search heuristic algorithm for solving optimization problems. Therefore, this paper uses AGA to optimize the initial weights and thresholds of BP neural network, so that AGA-BP neural network model has better prediction accuracy. Genetic algorithm optimizes the algorithm flow of three-layer BP network connecting weights and thresholds: the network weights and thresholds are arranged in a certain order into a real number array, which is used as the chromosome group of genetic algorithm. The fitness of each chromosome was calculated. According to a certain probability of crossover and mutation, new individuals are generated, and whether they meet the optimization criteria or the number of cycles is judged. If the number of cycles is less than the number of networks, the number of hidden nodes in the network is changed to recalculate. If the number of cycles is consistent, the best individuals are saved and compared, and the optimal individuals are selected. The initial weights and thresholds of BP network are obtained by splitting the optimal individuals in order [9]. 


\subsection{Principal Component Analysis}

Using principal component analysis (PCA) to reduce the dimension of data samples can make the subsequent diagnosis and analysis more efficient and convenient. In fact, PCA uses similar functions to transform the data set of multiple variables into a data set with fewer new variables (i.e., principal components), and the less new variables contain most of the information of the original data set. The function to calculate the similarity between two adjacent groups of data is;

$$
\begin{aligned}
& R_{i j}=\exp \left(-\frac{1}{\delta}\left|x_{i}-x_{j}\right|^{2}\right) \\
& \sigma=\sum_{i=1}^{\mathrm{n}}\left(\max x_{i}-\min x_{j}\right)
\end{aligned}
$$

Where $x_{\mathrm{i}}$ and $x_{\mathrm{j}}$ are the adjacent two groups of data; $R_{\mathrm{ij}}$ is the similarity of two adjacent groups of data; $\delta$ is the normalization parameter. If $R_{\mathrm{ij}}<\lambda(\lambda<1)$, keep two groups of data, otherwise delete a group of data, so as to reduce the data input of neural network and avoid data disaster [10].

\section{Experimental Design}

\subsection{Experimental Data}

The vibration data in this paper are obtained from the gearbox fault diagnosis experiment published by Jiangsu qianpeng Diagnostic Engineering Co., Ltd. The vibration data of the gearbox input shaft bearing at $880 \mathrm{r} / \mathrm{min}$ motor speed is selected. The sampling frequency of the data is $5120 \mathrm{~Hz}$. The sampling time is 10 seconds, i.e. 51200 sampling points for each state of the original vibration data. According to relevant research statistics, $60 \%$ of the faults in the actual work of the gearbox occur in the gear failure, and the fault forms of the gear mainly include tooth pitting, tooth breakage and tooth wear. Therefore, the vibration data of the gear box high-speed shaft in normal state, pitting state, broken tooth state and wear state are selected for processing and analysis.

\subsection{Eigenvalue Extraction}

The commonly used time-domain index parameters mainly include dimensional parameters and dimensionless parameters. Each parameter category contains several indicators. Different indicators can reflect different characteristics of signals, and can be used to determine whether mechanical equipment has faults, fault development degree and trend [11]. Frequency domain analysis can show the signal characteristics clearly, which can reflect the fault type and position of gearbox. At the same time, some other commonly used feature indexes, such as information entropy and fractal dimension, can be extracted. 
Considering the complexity and instability of vibration signal, wavelet entropy is selected in information entropy, and 10 indexes are selected in time domain, such as mean and skewness. In the frequency domain, three indexes are selected, i.e. gravity center frequency, correlation factor and frequency-domain mean square error, and box dimension is used in fractal dimension. That is, a group of samples is composed of these 15 characteristic indexes, that is to say, the dimension of the input sample is 15 dimensions.

The selected original vibration data are divided into 25 groups, and each type is labeled with 1 to 25 groups. Among them, 1 to 20 groups of data are used as training samples of AGA-BP neural network, and 21-25 groups are used as test samples. Therefore, there are 80 training sets and 20 test sets. The characteristic values of some samples are shown in Table 1.

The selected original vibration data are divided into 25 groups, and each type is divided into 25 groups, with 1-20 groups as training samples and 21-25 groups as test samples. Therefore, there are 80 training sets and 20 test sets. The characteristic values of some samples are shown in Table 1.

Table 1. Partial feature set sample

\begin{tabular}{crrrr}
\hline Characteristic index & Normal 1 & Pitting 1 & Broken teeth 1 & Abrasion 1 \\
\hline Wavelet entropy & & & & 2.442 \\
Box dimension & 1.640 & 1.899 & 1.027 & 1.323 \\
mean value & 1.219 & 1.192 & 1.294 & 4.321 \\
peak value & 3.344 & 3.180 & 3.359 & 36.622 \\
Kurtosis & 15.870 & 18.921 & 23.347 & 12.179 \\
Skewness & 3.247 & 4.372 & 8.480 & 0.139 \\
Peak index & -0.084 & -0.177 & 0.157 & 6.077 \\
Pulse index & 3.895 & 4.753 & 5.514 & 8.475 \\
Waveform index & 4.746 & 5.950 & 6.951 & 1.395 \\
clearance factor & 1.218 & 1.252 & 1.260 & 10.327 \\
kurtosis value & 5.533 & 7.031 & 8.153 & 0.009 \\
Skewness index & 0.012 & 0.017 & 0.026 & 0.001
\end{tabular}

According to table 2, the contribution rate of the first component is $59.4922 \%$, and the eigenvalue is 10.1137 , which is absolutely dominant. The cumulative contribution rate of the first five components is $94.9454 \%$, which means that the first five components can well reflect most of the information of the original characteristic matrix. In principal component analysis, the principal components whose cumulative contribution rate reaches $85 \%$ to $95 \%$ are generally selected, so the first five components are the training set and test set of neural network. 
Table 2. Principal component contribution rate

\begin{tabular}{ccccc}
\hline component & characteristic value & difference & contribution rate\% & $\begin{array}{c}\text { cumulative } \\
\text { contribution rate\% }\end{array}$ \\
\hline 1 & 10.1137 & 7.2766 & 59.4922 & 59.4922 \\
2 & 2.8371 & 1.0818 & 16.6888 & 76.1810 \\
3 & 1.7553 & 0.7300 & 10.3251 & 86.5061 \\
4 & 1.0253 & 0.6159 & 6.0311 & 92.5371 \\
5 & 0.4094 & 0.0180 & 2.4083 & 94.9454 \\
\hline
\end{tabular}

\subsection{Establishment of AGA-BP Neural Network Model}

For the setting of parameters of AGA-BP neural network model, according to each group of data samples used, there are 5 independent variables, so the number of input layer neural nodes is 5 , and the output layer is 4 , which corresponds to the four states of gearbox; normal state, gear pitting, gear broken, gear wear.

For the number of hidden layer neural nodes, refer to empirical formula (5), where $\mathrm{n}$ is the number of neural nodes in the input layer of neural network, i.e., $\mathrm{n}=5$. $\mathrm{M}$ is the number of neural nodes in the output layer of AGA-BP neural network, that is, $\mathrm{M}=4$, and $\mathrm{B}$ is a real number from 1 to 10 . After many experiments, the number of hidden layer nodes is set to 9 .

$$
\mathrm{M}=\sqrt{m+n}+b
$$

The structure of the neural network model is $\mathrm{n}(5,9,4)$. For the selection of neural network transfer function, Tansig function is selected from input layer to hidden layer and hidden layer to output layer, while trainlm function is selected as training function by default. The learning rate of the neural network model is set to 0.1 , the expected error is 0.001 , and the maximum training times are 1000. For the parameter setting of genetic algorithm, the initial population is 40 , and the maximum number of iterations is 150.

For the parameter setting of genetic algorithm, the initial population is 40 , and the maximum number of iterations is 150 .After 45 iterations of the training algorithm, the error is 0.000192 , which shows the effectiveness of the AGA-BP neural network model for gearbox fault diagnosis. AGA and SGA are used to optimize BP neural network, as shown in Figure 2. After 100 iterations of AGA fitness value, the fitness decreases slowly, and reaches the optimal fitness at about 140 times. It can be seen that the optimal fitness value of AGA is much smaller than that of SGA. The smaller the fitness is, the better the optimization effect is.

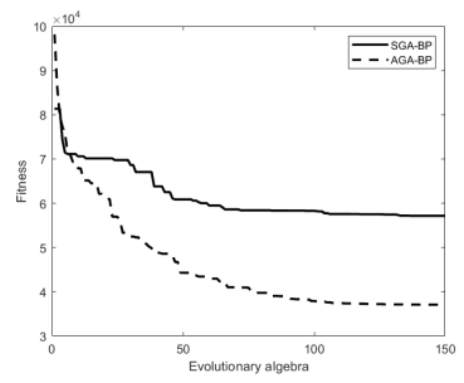

Figure 2. Fitness curve of SGA-BP and AGA-BP 


\section{Experimental Result}

After normalization, 21 to 25 groups of extracted feature sets are used as test samples. After normalization, they are put into the trained AGA-BP neural network. The target vectors representing the four states of gearbox are: normal state $T_{1}=(1,0,0,0)$; gear pitting $T_{2}=(0,1,0,0)$; gear broken teeth $T_{3}=(0,0,1,0)$; gear wear $T_{4}=(0,0,0,1)$. The target vector is $T=\left(T_{1}, T_{2}, T_{3}, T_{4}\right)$. The recognition results are obtained, as shown in Table 3. It can be seen from the diagnosis results that only the 25th group error of pitting corrosion state is relatively large, the other groups are within the allowable range of error, and each group of errors are relatively small, and the recognition accuracy is relatively high. MSE error of AGA-BP neural network is 0.0116. The smaller the mean square error (MSE) is, the better the prediction model is to describe the experimental data.

Table.3. Diagnosis results of AGA-BP neural network

\begin{tabular}{|c|c|c|c|c|c|}
\hline \multirow{2}{*}{$\frac{\text { Test data }}{\text { Normal } 21}$} & \multicolumn{4}{|c|}{ AGA-BP diagnosis results } & \multirow[t]{2}{*}{ Target vector } \\
\hline & 1.0023 & 0.0034 & 0.0004 & -0.0003 & \\
\hline Normal 22 & 1.0032 & 0.0012 & 0.0010 & -0.0006 & \\
\hline Normal 23 & 1.0049 & 0.0017 & 0.0017 & -0.0016 & 1000 \\
\hline Normal 24 & 1.0038 & 0.0010 & -0.0005 & 0.0009 & \\
\hline Normal 25 & 1.0012 & 0.0015 & -0.0021 & 0.0027 & \\
\hline Pitting 21 & 0.0034 & 1.0001 & -0.0005 & 0.0019 & \\
\hline Pitting 22 & 0.0018 & 0.9991 & -0.0003 & 0.0020 & \\
\hline Pitting 23 & 0.0044 & 0.9987 & 0.0016 & -0.0010 & 0100 \\
\hline Pitting 24 & 0.0046 & 0.9979 & 0.0003 & 0.0006 & \\
\hline Pitting 25 & 0.1243 & 0.8784 & -0.0017 & 0.0029 & \\
\hline Broken teeth 21 & 0.0000 & 0.0021 & 0.9877 & 0.0126 & \\
\hline Broken teeth 22 & -0.0055 & 0.0086 & 0.9720 & 0.0251 & \\
\hline Broken teeth 23 & -0.0015 & 0.0010 & 0.9893 & 0.0102 & 0010 \\
\hline Broken teeth 24 & 0.0016 & 0.0013 & 0.9832 & 0.0160 & \\
\hline Broken teeth 25 & 0.0023 & 0.0006 & 0.9973 & 0.0015 & \\
\hline Abrasion 21 & -0.0003 & -0.0006 & 0.0021 & 0.9971 & \\
\hline Abrasion 22 & 0.0003 & 0.0006 & 0.0005 & 0.9991 & \\
\hline Abrasion 23 & 0.6681 & -0.6654 & 0.0230 & 0.9732 & 00001 \\
\hline Abrasion 24 & -0.0233 & 0.0143 & 0.0379 & 0.9739 & \\
\hline Abrasion 25 & -0.0001 & 0.0017 & -0.0015 & 1.0007 & \\
\hline
\end{tabular}

\section{Conclusion}

Based on the characteristics of gearbox vibration signal, a gearbox fault diagnosis method based on multi eigenvalue extraction, PCA and AGA-BP neural network analysis is proposed and verified in this paper. The MSE of 20 groups of test samples is 0.0116 , which improves the accuracy of gearbox fault diagnosis, and the recognition rate of fault diagnosis is $100 \%$. It is verified that the network model can be used for 
gearbox fault diagnosis, which provides a more reliable method for high-speed train gearbox fault diagnosis.

\section{References}

[1] Shao RP, Xue T, Ma J. Study on vibration and acoustic radiation characteristics of reducer system. Computer Measurement and Control, 2011,19 (9):22-25.

[2] Wang YS. Research on theory and application technology of condition monitoring. Signal Analysis and Fault Diagnosis of Steam Turbine Generator Set. Nan Jing:Southeast University, 2000.

[3] Wang YZ, Wu CH, Zhou DQ, et al. Research on photovoltaic array fault diagnosis based on BP neural network. Power System Protection and Control, 2013,41(16):108-114.

[4] Zhang SH, Ou JP. BP-PSO based intelligent case retrieval method for high-rise structural form selection. Science China Technological Sciences, 2013,56(4):940-994.

[5] Yang J, Li YH, Xiong DS. Fault diagnosis of induction motor based on wavelet packet energy spectrum and improved neural network. Power Electronics, 2013,11(2):19-21.

[6] Zhou P. Matlab design and application of neural network. Bei Jing:Tsinghua University Press:2013.

[7] Fardin A, Khabat S, Fardin AT, et al. Artificial neural network development by means of a novel combination of grammatical evolution and genetic algorithm. Engineering Applications of Artificial Intelligence, 2015, 39:1-13.

[8] Chen XL, Wang K, Peng JS, et al. Holiday traffic flow forecast based on improved GA-BP algorithm. Highway Traffic Technology, 2018,34(06):114-117.

[9] Zhang X, Guo X, Yu YH, et al.A fault diagnosis method based on BP neural network optimized by genetic algorithm. Innovation and Application of Science and Technology, 2019(29):15-17.

[10] Chen Y, Li P. Online forecasting model of transmission line icing load based on PCA-GA-LSSVM. Power System Protection and Control, 2019,47(10):110-119.

[11] Lei JR, et al. Engineering Technology Processing Technology. Chong Qing:Chongqing University Press, 1990 . 